\title{
DISCRETE SPECTRUM OF THE WEIL REPRESENTATION
}

\author{
BY S. RALLIS AND G. SCHIFFMANN
}

Communicated by J. A. Wolf, September 10, 1976

1. Weil representation. Let $Q$ be a nondegenerate quadratic form on $\mathbf{R}^{k}$. Let $O(Q)$ be the orthogonal group of $Q$. One owes to A. Weil [4] the construction of a certain unitary representation $\pi_{Q}$ of the group $\widetilde{\mathrm{Sl}_{2}} \times O(Q)$ in $L^{2}\left(\mathbf{R}^{k}\right)$, where $\widetilde{\mathrm{Sl}_{2}}$ is a two fold covering of $\mathrm{Sl}_{2}(\mathbf{R})$, i.e. given by pairs $(g, \epsilon)$ with $g \in$ $\mathrm{Sl}_{2}(R)$ and $\epsilon= \pm 1$ satisfying the group law $(g, \epsilon)\left(g^{\prime}, \epsilon^{\prime}\right)=\left(g g^{\prime}, V\left(g, g^{\prime}\right) \epsilon \epsilon^{\prime}\right)$, where $V$ is the Kubota cocycle on $\mathrm{Sl}_{2}(\mathbf{R})$ (with values in $\mathrm{Z}_{2}$ ). Let $w_{0} \in \widetilde{\mathrm{SI}_{2}}$ be the element $\left(\left[\begin{array}{rr}0 & -1 \\ 1 & 0\end{array}\right],-1\right)$. Then $\pi_{Q}$ is given by

$$
\pi_{Q}\left(w_{0}\right) \varphi(X)=\delta_{Q} \hat{\varphi}\left(-M_{Q}(X)\right), \varphi \in L^{2}\left(\mathbf{R}^{k}\right)
$$

where $M_{Q} \in \operatorname{Aut}\left(\mathbf{R}^{k}\right)$ so that $\left[X, M_{Q}(Y)\right]=Q(X, Y)$ for all $X, Y \in \mathbf{R}^{k}$ (with [, ] the usual dot product on $\mathrm{R}^{k}$ ) and $\delta_{Q}=|\operatorname{det} Q|^{-1 / 2} u_{Q}$ with $u_{Q}$ a certain eighth root of unity determined explicitly in [2]. Moreover, $\wedge$ denotes the Fourier transform on $L^{2}\left(\mathbf{R}^{k}\right)$. Also we have

$$
\pi_{Q}\left(\left[\begin{array}{ll}
\alpha & \beta \\
0 & \alpha^{-1}
\end{array}\right], 1\right) \varphi(X)=|\alpha|^{k / 2} e^{\sqrt{-1} \pi \beta \alpha Q(X, X)} \varphi(\alpha X) \text {, with } \alpha>0
$$

and

$$
\pi_{Q}(g) \varphi(X)=\varphi\left(g^{-1} X\right) \text { for } g \in O(Q) .
$$

Then (i), (ii), and (iii) determine $\pi_{Q}$ explicitly. The main problem is to give a spectral decomposition of $\pi_{Q}$.

2. Discrete spectrum of $\pi_{Q}$. Let $\widetilde{K}$ be the maximal compact subgroup of $\widetilde{\mathrm{Sl}_{2}}$ given by

$$
\left\{\left(\left[\begin{array}{cc}
\cos \theta & \sin \theta \\
-\sin \theta & \cos \theta
\end{array}\right], \epsilon\right) \mid-\pi \leqslant \theta<\pi, \epsilon= \pm 1\right\} .
$$

Then every unitary character of $K$ is given by

$$
k(\theta, \epsilon) m(\operatorname{sgn} \epsilon)^{2 m} e^{\sqrt{-1} m \theta} \quad \text { with } m \in 1 / 2 Z
$$

We let

$$
A=\left\{a(r)=\left(\left[\begin{array}{ll}
r & 0 \\
0 & r^{-1}
\end{array}\right], 1\right) \mid r>0\right\}
$$

AMS (MOS) subject classifications (1970). Primary 22E45; Secondary 43A80. 
and

$$
N=\left\{n(x)=\left(\left[\begin{array}{ll}
1 & x \\
0 & 1
\end{array}\right], 1\right) \mid x \in \mathbf{R}\right\}
$$

Let $\mathrm{a}, \mathrm{n}$, and be the infinitesimal generators of $A, N$, and $K$, respectively. Then

$$
\omega_{\mathrm{Sl}_{2}}=-\mathfrak{t}^{2}+\mathfrak{a}^{2}+\left(\mathfrak{n}+\operatorname{Ad}\left(w_{0}\right) \mathfrak{n}\right)^{2}
$$

is the Casimir element of $\widetilde{\mathrm{Sl}_{2}}$. We let

$$
E_{+}=\mathfrak{l}+\sqrt{-1}\left(\mathfrak{n}+\operatorname{Ad}\left(w_{0}\right) \mathfrak{n}\right) \text { and } E_{-}=k-\sqrt{-1}\left(\mathfrak{n}+\operatorname{Ad}\left(w_{0}\right) \mathfrak{n}\right) .
$$

We assume that $Q$ has inertia type $(a, b)$ where $a \geqslant b \geqslant 1$ and $a+b=$ $k \geqslant 3$. Then we choose a splitting of $Q$ on $\mathbf{R}^{k}=\mathbf{R}^{a} \oplus \mathbf{R}^{b}$ so that $X=X_{+}+$ $X_{\text {_ with }} X_{+} \in \mathbf{R}^{a}, X_{-} \in \mathbf{R}^{b}$ and $Q(X, X)=\left\|X_{+}\right\|^{2}-\left\|X_{-}\right\|^{2}(\|\|=$ usual length of vector in $\mathbf{R}^{t}$ ).

We consider $\mathbf{F}_{Q}(\lambda)=\left\{\varphi \in \mathbf{F}_{Q} \mid \omega_{\mathrm{Sl}_{2}} \cdot \varphi=\lambda \varphi\right\}$ where $\mathbf{F}_{Q}$ is the space of $C^{\infty}$ vectors in $L^{2}\left(\mathbf{R}^{k}\right)$ of $\pi_{Q}$. Let $\Omega_{+}=\{X \mid Q(X, X)>0\}$ and $\Omega_{-}=$ $\{X \mid Q(X, X)<0\}$.

THEOREM 1. The spaces $\mathbf{F}_{Q}^{+}(\lambda)=\left\{\varphi \in \mathbf{F}_{Q}(\lambda)|\varphi|_{\Omega_{-}} \equiv 0\right\}$ and $\mathbf{F}_{Q}^{-}(\lambda)=$ $\left\{\varphi \in \mathrm{F}_{Q}(\lambda)|\varphi|_{\Omega_{+}} \equiv 0\right\}$ are $\widetilde{\mathrm{Sl}_{2}} \times O(Q)$ stable subspaces. Moreover, $\mathrm{F}_{Q}^{+}(\lambda)$ and $\mathbf{F}_{Q}^{-}(\lambda)$ (if nonzero) determine topologically irreducible representations of $\mathrm{SI}_{2} \times$ $O(Q)$ which are inequivalent. Also $\mathbf{F}_{Q}(\lambda)$ is the direct sum of $\mathbf{F}_{Q}^{+}(\lambda)$ and $\mathbf{F}_{Q}^{-}(\lambda)$.

We let

$$
\begin{aligned}
& L^{2}(\text { Whit })=\left\{f: \widetilde{\mathrm{Sl}_{2}} \rightarrow \mathrm{C} \mid f(g n(x))=f(g) e^{2 \pi \sqrt{-1} x}\right. \\
& \left.\qquad \text { for all } g \in \widetilde{\mathrm{Sl}_{2}}, x \in \mathbf{R} \text { and } \int_{\widetilde{\mathrm{SI}_{2} / N}}|f(g)|^{2} d \mu(g)<\infty\right\},
\end{aligned}
$$

where $d \mu$ is an $\widetilde{\mathrm{SI}_{2}}$ invariant measure on $\widetilde{\mathrm{SI}_{2}} / N$. We consider the subspace $L^{2}$ (Whit) $\left.)_{\lambda}=\left\{\psi \in L^{2} \text { (Whit) }\right)_{\infty} \mid \omega_{\mathrm{Sl}_{2}} * \psi=\lambda \psi\right\}$. (( ) denotes $C^{\infty}$ vectors of representation.)

THEOREM 2. The discrete spectrum of $L^{2}$ (Whit) $)_{\infty}$ is the direct sum $\bigoplus_{s \in \tilde{A}} L^{2}$ (Whit) $s^{2}-2 s$, where $\widetilde{A}=\{1 / 2 m>0 \mid m \in \mathbf{Z}\}$. Moreover, each $L^{2}$ (Whit) ${ }_{s^{2}-2 s}$ is $\overline{\mathrm{S}}_{2}{ }^{2}$ irreducible and corresponds to a square integrable representation of ${\overline{\mathrm{ST}_{2}}}_{2}^{2 s}$.

("Discrete spectrum" means the sum of all those irreducible representations of $\widetilde{\mathrm{SI}_{2}}$ which occur discretely in $L^{2}$ (Whit).) 
THEOREM 3. The space $\mathbf{F}_{Q}^{+}(\lambda) \neq 0$ if and only if $\lambda=s^{2}-2 s$ with $s \in$ $\widetilde{A}-\{1 / 2\}$ and $s \equiv 1 / 2 k$ mod 1. The representation of $\widetilde{\mathrm{SI}_{2}} \times O(Q)$ in $\mathbf{F}_{Q}^{+}\left(s^{2}-2 s\right)$ is equivalent to the tensor product of $L^{2}$ (Whit) $s^{2}-2 s \hat{\otimes} A_{s}^{+}$, where $A_{s}^{+}=$ $\left\{\varphi \in \mathbf{F}_{Q} \mid \mathfrak{t} \cdot \varphi=\sqrt{-1}\right.$ s $\varphi$ and $\left.E_{+} \varphi=0\right\}$. Moreover, $A_{s}^{+}$is an irreducible $O(Q)$ module.

We note that for the case $k=3$ an analogous tensor product as in Theorem 3 is discussed in [1].

REMARK 1. If $b=1$, then $\mathbf{F}_{Q}^{-}(\lambda)=0$ for all $\lambda$. And if $b>1$, then as in Theorem $2, \mathbf{F}_{Q}^{-}(\lambda) \neq 0$ if and only if $\lambda=s^{2}-2 s$ with $s \in \widetilde{A}-\{1 / 2\}$ and $s \equiv$ $1 / 2 k$ mod 1. Similarly $F_{Q}^{-}\left(s^{2}-2 s\right)$ is $\widetilde{\mathrm{Sl}_{2}} \times O(Q)$ equivalent to the tensor product $L^{2}$ (Whit)* ${ }_{s^{2}-2 s}^{*} \hat{\otimes} A_{-s}^{-}$, with $L^{2}$ (Whit) ${ }_{s^{2}-2 s}$ the representation of $\widetilde{\mathrm{ST}_{2}}$ in $L^{2}$ (Whit) $s^{2}-2 s$ after conjugation by the unique outer automorphism of $\widetilde{\mathrm{St}_{2}}$, and $A_{-s}^{-}=\left\{\varphi \in \mathbf{F}_{Q} \mid \ell \varphi=-\sqrt{-1} s \varphi, E_{-}(\varphi)=0\right\}$.

Then the space $A_{s}^{+}$is characterized in several ways.

THEOREM 4. $A_{s}^{+}$is $O(Q)$ equivalent to the representation of $O(Q)$ in the spaces $\left\{\beta \in L^{2}\left(\Gamma_{1}\right)_{\infty} \mid W_{\xi}^{+} * \beta=\left(s^{2}-2 s+k-1 / 4 k^{2}\right) \beta\right\}$ where $\Gamma_{1}$ is the hyperboloid $\left\{X \in \mathbf{R}^{k} \mid Q(X, X)=1\right\}$ and $W_{\xi}^{+}$the Laplace Beltrami operator on $\Gamma_{1}$ determined by the separation of variables of

$$
\partial(Q)=\frac{\partial^{2}}{\partial t^{2}}+\frac{k-1}{t} \frac{\partial}{\partial t}-\frac{1}{t^{2}} W_{\xi}^{+}
$$

(with $X=t \cdot \xi, \xi \in \Gamma_{1}$ ).

REMARK 2. We note here results on the discrete spectrum of the hyperboloid similar to Theorem 4 are obtained in [3] in a different framework.

We let $K$ be the maximal compact subgroup of $O(Q)$. Then $K$ is isomorphic to the product $O(a) \times O(b)$, where $O(t)$ is the standard orthogonal group in $t$ variables. We consider the family of irreducible representations $\left[s_{1}\right]_{a} \otimes\left[s_{2}\right]_{b}$ of $K$, where $[x]_{t}$ denotes the representation of $O(t)$ on spherical harmonics of degree $t$. Then let $E_{Q}\left(s^{2}-2 s, m, s_{1}, s_{2}\right)$ be the $\widetilde{K} \times K$ isotypic component in $\mathbf{F}_{Q}^{+}\left(s^{2}-2 s\right)$ which transforms according to the character

$$
k(\theta, \epsilon) m(\operatorname{sgn} \epsilon)^{2 m} e^{\sqrt{-1} \theta m}
$$

on $\widetilde{K}$ and according to $\left[s_{1}\right]_{a} \otimes\left[s_{2}\right]_{b}$ on $K$.

THEOREM 5. The space of $\widetilde{K} \times K$ finite vectors in $\mathbf{F}_{Q}^{+}\left(s^{2}-2 s\right)$ is the direct sum of the $E_{Q}\left(s^{2}-2 s, m, s_{1}, s_{2}\right)$, where $m=s+2 j, j$ a nonnegative integer and $s_{1}$ and $s_{2}$ satisfy the relation $s_{1}-s_{2}=s-1 / 2(a-b)+2 j$. Moreover, each space $E_{Q}\left(s^{2}-2 s, s+2 j, s_{1}, s_{2}\right)$ is spanned by elements of the form (determined on $\Omega_{+}$) 


$$
\begin{array}{r}
\psi_{s, j}(Q(X, X)) Q(X, X)^{s-1} e^{-\pi Q(X, X)}\left\|X_{-}\right\|^{s_{2}}\left\|X_{+}\right\|^{-\left(s+k / 2+s_{2}-2\right)} \\
{ }_{2} F_{1}\left(\frac{1}{2}\left(s+s_{1}+s_{2}\right)+\frac{1}{4} k-1,-j, s_{2}+\frac{1}{2} b,\left(\frac{\left\|X_{-}\right\|}{\left\|X_{+}\right\|}\right)^{2}\right) \\
\cdot P_{s_{1}}\left(\frac{X_{+}}{\left\|X_{+}\right\|}\right) P_{s_{2}}\left(\frac{X_{-}}{\left\|X_{-}\right\|}\right),
\end{array}
$$

where ${ }_{2} F_{1}$ is the usual hypergeometric function, $P_{s_{1}}$ and $P_{s_{2}}$ are harmonic polynomials of degree $s_{1}$ and degree $s_{2}$ in $\mathbf{R}^{a}$ and $\mathbf{R}^{b}$, respectively, and $\psi_{s, j}(u)$ is the polynomial $\Sigma_{\nu=0}^{\nu=j} c_{\nu} u^{j-\nu}$ with

$$
c_{\nu}=\frac{(-1)^{\nu}}{2^{\nu} \nu !} \frac{\Gamma(s+j)}{\Gamma(s+j-\nu)} \frac{j !}{(j-\nu) !} .
$$

As an important consequence of Theorem 5 we deduce growth and continuity properties of $\widetilde{K} \times K$ finite vectors in $\mathrm{F}_{Q}^{+}\left(s^{2}-2 s\right)$.

Corollary to Theorem 5. Every $\widetilde{K} \times K$ finite function $\varphi$ in $\mathbf{F}_{Q}^{+}\left(s^{2}-2 s\right)$ extends uniquely to a continuous function on $\mathbf{R}^{k}-\{0\}$ which vanishes identically on $\left(\Omega_{-} \cup \Gamma_{0}\right)-\{0\}$. Moreover, if $s>1 / 2 k$, then $\varphi$ extends uniquely to a continuous function on $\mathbf{R}^{k}$ which vanishes identically on $\Omega_{-} \cup \Gamma_{0} . A l s o$ such a $\varphi$ satisfies the Poisson Summation Formula Property, that is, for any lattice $L \subseteq \mathbf{R}^{k}$ with $Q(L, L) \subseteq \mathbf{Z}$, the integers,

$$
F(X)=\sum_{\xi \in L} \varphi(X+\xi),
$$

is continuous (with the summation satisfying absolute convergence) on $\mathbf{R}^{k}$ and $\Sigma_{\xi * \in L} \hat{\varphi}\left(\xi^{*}\right)$ is absolutely convergent ( $L^{*}$ dual lattice to $L$ ).

We remark that similar types of statements hold for $\widetilde{K} \times K$ functions $f \in \mathbf{F}_{Q}^{-}\left(s^{2}-2 s\right)$.

\section{BIBLIOGRAPHY}

1. S. Gelbart, Weil's representation and the spectrum of the metaplectic group, Lecture Notes in Math., no. 530, Springer-Verlag, Berlin and New York, 1976.

2. S. Rallis and G. Schiffmann, Weil representation. I: Intertwining distributions and discrete spectrum, 1975 (preprint).

3. R. Strichartz, Harmonic analysis on hyperboloids, J. Functional Analysis 12 (1973), 341-383. MR 50 \#5370.

4. A. Weil, Sur certains groupes d'operateurs unitaires, Acta Math. 111 (1964), 143-211. MR 29 \#2324.

\section{DEPARTMENT OF MATHEMATICS, UNIVERSITY OF NOTRE DAME, NOTRE DAME, INDIANA 46556}

INSTITUT DE RECHERCHE MATHÉMATIQUE AVANCÉE, UNIVERSITÉ LOUIS PASTEUR, 7, RUE RENÉ DESCARTES, 67084-STRASBOURG, CEDEX, FRANCE (Current address of G. Schiffmann)

Current address (Stephen Rallis): Department of Mathematics, Princeton University, Princeton, New Jersey 08540 